month follow-up. Eye. 2019. https://doi.org/10.1038/s41433-0180323-9. Epub ahead of print.

2. Wu W, Wang Y, Xu L. Meta-analysis of Pentacam vs. ultrasound pachymetry in central corneal thickness measurement in normal, post-LASIK or PRK, and keratoconic or keratoconussuspect eyes. Graefes Arch Clin Exp Ophthalmol. 2014;252: 91-9.
3. Cavas-Martínez F, Bataille L, Fernández-Pacheco DG, Cañavate FJF, Alio JL. Keratoconus detection based on a new corneal volumetric analysis. Sci Rep. 2017;7:15837.

4. O'Brart DPS. Corneal collagen crosslinking for corneal ectasias: a review. Eur J Ophthalmol. 2017;27:253-69.

5. Hashemi H, Seyedian MA, Miraftab M, Fotouhi A, Asgari S. Corneal collagen cross-linking with riboflavin and ultraviolet A irradiation for keratoconus: long-term results. Ophthalmology. 2013;120:1515-20.

\title{
Management of the photic sneeze reflex utilising the philtral pressure technique
}

\author{
Samantha Bobba' ${ }^{1}$ Sascha K. R. Spencer ${ }^{2}$ - Olivia J. K. Fox ${ }^{3} \cdot$ Ashish Agar $^{2,4} \cdot$ Minas T. Coroneo $^{2,4} \cdot$ lan C. Francis ${ }^{2,4}$
}

Received: 16 October 2018 / Revised: 13 January 2019 / Accepted: 22 January 2019 / Published online: 19 February 2019

(c) The Royal College of Ophthalmologists 2019

\section{The photic sneeze reflex}

The Photic Sneeze Reflex (PSR) is a neuro-ophthalmological phenomenon, consisting of sneezing in response to an external light stimulus. The PSR, aka Autosomal dominant Compelling Helio-Ophthalmic Outburst (ACHOO) syndrome, was first described by Aristotle in 350 BC [1]. PSR reportedly occurs secondary to a change in light intensity, typically at onset of light exposure, and increases with lacrimation or nasal irritability [2].

The pathophysiology of the PSR has not been clearly elucidated. The sneeze, or sternutatory reflex, involves an afferent arc from the upper anterior nose through the anterior ethmoidal branch of the ophthalmic division of the trigeminal nerve, and from the lower nose and orbit via the maxillary division $[2,3]$. One proposed mechanism of the PSR includes optic-trigeminal summation in which persistent light exposure relaying signals via the optic and trigeminal nerves may lead to increased sensitivity in the

Samantha Bobba

samantha.bobba@gmail.com

$\triangle$ Ian C. Francis

iancfrancis@gmail.com

1 Westmead Hospital, Westmead, Sydney, Australia

2 University of New South Wales, Kensington, Sydney, Australia

Nepean Hospital, Nepean, Sydney, Australia

4 Department of Ophthalmology, Prince of Wales Hospital, Randwick, Sydney, Australia maxillary rather than ophthalmic branch, resulting in a sneeze rather than photophobia. Alternatively, ocular sensory input could lead to activation of neighbouring neurons involved in the sneeze response due to parasympathetic generalisation [2].

There is no recognised management for PSR. A military report demonstrated that interference filters were ineffective, suggesting that PSR appears to be mediated by changes in light intensity, rather than wavelength [3].

When examining patients on the slit lamp, PSR may be an unpredictable challenge to the physical integrity of the patient-doctor relationship. For the Neurologist who may have his/her face in extreme proximity to the patient's face when using the direct ophthalmoscope, the PSR may prove infectious. Sneezing has also been reported to result in rib fractures in osteoporotic patients, spontaneous abortion, ruptured intracranial aneurysm, aortic dissection, intervertebral disc prolapse leading to quadriplegia, and death $[4,5]$.

Over 35 years, six patients who demonstrated PSR were examined in a suburban Ophthalmological practice. Five patients were males aged 25 to 81, with PSR for 6-20 years, and the sixth patient was an eight-week old female.

One patient offered a practical approach to minimising the PSR during clinical examination by utilising the Philtral Pressure Technique (PPT). This involved firm digital pressure applied by the patient's index finger transversely to the skin of the sub-philtral region, directed posterosuperiorly onto the maxilla (Fig. 1). This was successful in preventing 

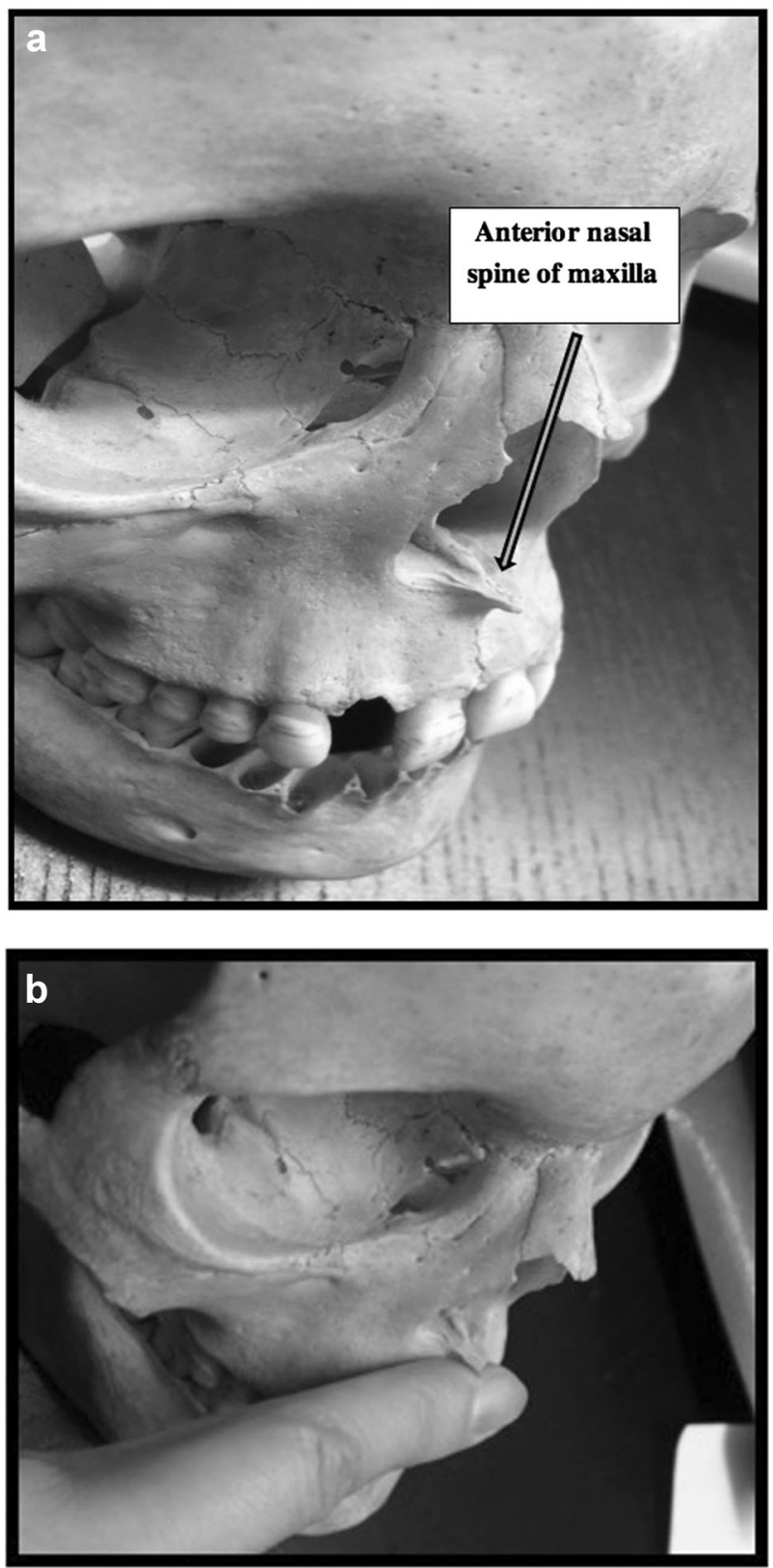

Fig. 1 a, b The anatomy and physiology of the PPT. Mechanism: Firm transverse pressure applied to the skin of sub-philtral region, directed posterosuperiorly onto the bone, and towards the anterior superior alveolar branch of the infraorbital nerve the PSR in three patients in whom it was necessary for clinical management.

One possible mechanism of the PPT is stimulation of local mechanoreceptors that may override the trigeminal nerve irritation. Alternatively, it may interfere with the coactivation of neighbouring parasympathetic fibres, preventing the PSR. The PPT may act as a 'sensory trick' (geste antagoniste), analogous to rubbing of the eyelid to reduce Benign Essential Blepharospasm.

PSR may prove a challenge in undertaking neuroophthalmological examinations. To date, there is no recognised management protocol for PSR in the clinical environment. The PPT technique was offered as a solution by one of the patients reported in this case series. PPT in the management of PSR remains to be tested in clinical trials.

\section{Compliance with ethical standards}

Conflict of interest: The authors declare that they have no conflict of interest.

Publisher's note: Springer Nature remains neutral with regard to jurisdictional claims in published maps and institutional affiliations.

\section{References}

1. Aristotle. Problem 33: 7. In: Rosner F, ed. Julius Preuss' Biblical and Talmudic medicine. New York: New York Hebrew Publishing Co, 1978:74-76.

2. Whitman BW, Packer RJ. The photic sneeze reflex: literature review and discussion. Neurology. 1993;43:868-71.

3. Breitenbach RA, Swisher RP, Kim MK, Patel BS. The photic sneeze reflex as a risk factor to combat pilots. Mil Med. 1993;158:806-9.

4. Abramson DC. Sudden unexpected sneezing during the insertion of peribulbar block under propofol sedation. Can J Anaesth. 1995;42:740-3.

5. Upadhyaya SGN, Large A A. Sneeze: an unusual trigger for aortic dissection. BMJ Case Rep. 2013; https://doi.org/10.1136/bcr-2013200619 